\title{
Un résumé de la surveillance, de la morbidité et de la microbiologie des cas confirmés en laboratoire de botulisme infantile au Canada, de 1979 à 2019
}

\author{
Richard Harris ${ }^{1}$, Christine Tchao ${ }^{2}$, Natalie Prystajecky², ${ }^{2}$ Jennifer Cutler ${ }^{4}$, John W Austin ${ }^{1 \star}$
}

\section{Résumé}

Contexte : Le botulisme infantile est une maladie toxicoinfectieuse rare causée par la colonisation de l'intestin du nourrisson avec la bactérie clostridia productrice de neurotoxines botuliques (i.e. Clostridium botulinum ou les souches neurotoxiques de $C$. butyricum ou de C. baratii). Notre objectif était d'examiner les données provenant de cas confirmés en laboratoire de botulisme infantile signalés au Canada afin de résumer l'incidence au fil du temps, en fonction de la répartition géographique par province ou territoire et par sexe, et de comparer ces paramètres avec les données du Système canadien de surveillance des maladies à déclaration obligatoire (SCSMDO). L'âge moyen de l'apparition des symptômes, le sérotype de la neurotoxine botulique (NTBo), les résultats du cas, la durée de l'hospitalisation et la pertinence des échantillons cliniques pour la confirmation en laboratoire ont également été déterminés.

Méthodes : Nous avons examiné les dossiers de laboratoire du Service de référence sur le botulisme de Santé Canada et du laboratoire de santé publique du Centre de contrôle des maladies de la Colombie-Britannique. La Base de données sur les congés des patients (BDCP) et la Base de données sur la morbidité hospitalière (BDMH) de l'Institut canadien d'information sur la santé (ICIS) ont été interrogées pour obtenir des données sur les hospitalisations pour des cas de botulisme infantile. Le SCSMDO a été consulté pour obtenir des données sur les cas signalés de botulisme infantile.

Résultats : De 1979 à 2019, 63 cas confirmés en laboratoire de botulisme infantile ont été confirmés par le Service de référence sur le botulisme de Santé Canada et le laboratoire de santé publique du Centre de contrôle des maladies de la Colombie-Britannique pour un taux annuel de 4,30 cas par million de naissances vivantes. De 1983 à 2018, 57 cas de botulisme infantile ont été signalés au SCSMDO. Sur les 63 cas confirmés par les laboratoires de référence, l'âge médian de l'apparition des symptômes était de 16 semaines avec un intervalle de deux à 52 semaines. La majorité des cas étaient de type $A(76 \%)$ et $B(21 \%)$, avec des cas uniques de type $F$ et de type $A B$. Sur les 23 cas confirmés en laboratoire avec des dossiers hospitaliers jumelés, 13 ont été transférés à des soins spéciaux et huit ont eu besoin du soutien d'un respirateur; aucun décès n'a été signalé.

Conclusion : Les spores de C. botulinum sont présentes naturellement dans l'environnement, de sorte que le diagnostic de botulisme infantile ne nécessite pas d'antécédents d'exposition à des aliments à risque élevé comme le miel. Les échantillons de selle sont les échantillons de diagnostic les plus utiles.
Cette oeuvre est mise à la disposition selon les termes de la licence internationale Creative Commons Attribution 4.0

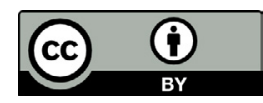

Affiliations

1 Service de référence pour le botulisme pour le Canada, Santé Canada, Ottawa, ON

${ }^{2}$ British Columbia Centre for Disease Control Public Health Laboratory, Vancouver, BC

${ }^{3}$ Department of Pathology and Laboratory Medicine, University of British Columbia, Vancouver, BC

${ }^{4}$ Agence de la santé publique du Canada, Guelph, ON

${ }^{\star}$ Correspondance : john.austin@canada.ca

Citation proposée : Harris R, Tchao C, Prystajecky N, Cutler J, Austin JW. Un résumé de la surveillance, de la morbidité et de la microbiologie des cas confirmés en laboratoire de botulisme infantile au Canada, de 1979 à 2019. Relevé des maladies transmissibles au Canada 2021;47(7/8):355-62.

https://doi.org/10.14745/ccdr.v47i78a05f

Mots-clés : Clostridium botulinum, Clostridium butyricum, Clostridium baratii, taux d'incidence, répartition géographique, Canada, sérotype, source environnementale, neurotoxine botulique 


\section{Introduction}

Le botulisme est une maladie neuroparalytique causée par l'exposition à la neurotoxine botulique (NTBo). Les manifestations de botulisme sont classées en fonction de la voie d'exposition aux NTBo. Le botulisme d'origine alimentaire est une intoxication résultant de l'ingestion de NTBo qui se trouve dans des aliments ou des boissons qui ont soutenu la croissance de Clostridium botulinum (1). Le botulisme par blessure souillée et le botulisme toxémique intestinal se produisent lorsque des spores de $C$. botulinum colonisent une blessure infectée ou le tractus intestinal des adultes, respectivement, et libèrent des NTBo concomitantes $(2,3)$.

Le botulisme infantile, décrit pour la première fois en $1976(4,5)$, est une forme de botulisme toxémique intestinal qui se produit chez les enfants de moins d'un an. Les nourrissons sont particulièrement sensibles à la colonisation intestinale par $C$. botulinum en raison de l'immaturité de leur microbiote intestinal, alors que les enfants de plus d'un an peuvent ingérer des spores de $C$. botulinum sans entraîner de colonisation $(6,7)$. Dans de rares cas, le botulisme infantile a été causé par les espèces productrices de NTBo, C. butyricum de type $E$ (8-10) et C. baratii de type $\mathrm{F}$ (11-14).

Le spectre clinique du botulisme infantile comprend un large éventail de gravités allant de l'infection subclinique à l'infection fulminante. Les symptômes peuvent inclure, sans toutefois s'y limiter, I'hypotonie, une succion faible, la dysphagie, la constipation, les faibles pleurs et la faiblesse du diaphragme qui peuvent exiger que le nourrisson reçoive une ventilation mécanique. Presque tous les cas de botulisme infantile sont causés par le $C$. botulinum du groupe I qui produit des NTBo de type $A$ ou $B$. Le type $A$ a, en général, une présentation clinique plus grave $(6,7)$.

Le botulisme infantile est traité au moyen de l'antitoxine sous forme d'immunoglobuline du botulisme administrée par intraveineuse (BIG-IV ou BabyBIG) qui se lie et neutralise les NTBo qui circulent dans le sang (15). Il est généralement recommandé que les nourrissons soient traités avec BabyBIG dès que possible en fonction du diagnostic différentiel d'un médecin. La confirmation en laboratoire du botulisme infantile est fondée sur la détection de $C$. botulinum viable dans les selles ou la détection de NTBo dans les selles ou le sérum.

Il y a eu peu de rapports publiés sur des cas de botulisme infantile au Canada (16-20). Nous présentons ici un résumé du botulisme infantile au Canada pour la période allant de 1979 à 2019, y compris l'incidence sur la période de déclaration, la répartition géographique par province et territoire, l'âge et le sexe du patient, le sérotype et le groupe de $C$. botulinum impliqués, la détermination des sources alimentaires ou environnementales (dans la mesure du possible), ainsi que le prélèvement des échantillons cliniques préférentiels pour la détection des NTBo et du C. botulinum. Les dossiers hospitaliers qui correspondent aux cas confirmés en laboratoire démontrent les résultats cliniques de la maladie.

\section{Méthodes}

\section{Laboratoire de microbiologie et données nationales de surveillance}

Nous avons examiné deux bases de données de laboratoire indépendantes pour les cas confirmés en laboratoire de botulisme infantile de 1979 à 2019. Ces bases de données sont tenues à jour par le Service de référence pour le botulisme pour le Canada de Santé Canada, à Ottawa, en Ontario, et le laboratoire de santé publique du Centre de contrôle des maladies de la Colombie-Britannique, à Vancouver, en Colombie-Britannique. Le Service de référence pour le botulisme de Santé Canada reçoit et analyse des échantillons cliniques et alimentaires associés à des cas suspectés de botulisme de la part de toutes les provinces et de tous les territoires, sur demande. Le laboratoire du Centre de contrôle des maladies de la Colombie-Britannique conserve sa capacité d'analyser des échantillons de la Colombie-Britannique et a également analysé des échantillons du Yukon. Ainsi, ces deux bases de données ne se chevauchent pas et, lorsqu'elles sont combinées, elles représentent tous les cas de botulisme confirmés en laboratoire au Canada.

Nous avons extrait des renseignements sur l'âge et le sexe des patients, la date et l'emplacement du diagnostic, la source impliquée de $C$. botulinum, le groupe de $C$. botulinum et le sérotype de NTBo. Les taux de maladies par million de naissances vivantes ont été calculés à l'aide des données de la Division de statistique de l'Organisation des Nations Unies (ONU) et de Statistique Canada $(21,22)$.

Nous avons utilisé la définition nationale des cas confirmés de botulisme infantile pour assurer l'uniformité de la consignation des données : " confirmation en laboratoire avec des symptômes compatibles avec le botulisme chez une personne de moins d'un an [avec] la détection de la toxine botulique dans les selles ou le sérum, ou l'isolement de $C$. botulinum dans les selles du patient ou au cours de l'autopsie » (23). Les cas répondant à cette définition ont été extraits du Système canadien de surveillance des maladies à déclaration obligatoire (SCSMDO) et comprenaient l'année de déclaration, la province ou le territoire, le groupe d'âge et le sexe. Le SCSMDO assure une surveillance de base des maladies à déclaration obligatoire à l'échelle nationale en recueillant des données fournies volontairement par les provinces et les territoires. Les données du SCSMDO ont été comparées aux données de laboratoire pour s'assurer qu'elles sont complètes. 


\section{Confirmation en laboratoire des cas cliniques}

La détection de la NTBo et l'isolement de $C$. botulinum viable dans des échantillons environnementaux et cliniques ont été effectués conformément à la méthode MFHPB-16 de Santé Canada (24). Le sérotype de NTBo a été déterminé en neutralisant la toxicité avec des anticorps spécifiques au sérotype fournis par les US Centers for Disease Control and Prevention. Le groupe de $C$. botulinum (groupe I ou II) a été déterminé en fonction de la protéolyse du milieu de viande cuite en culture pure (24). Le Département californien de la santé publique a identifié l'isolat solitaire de $C$. baratii de type $\mathrm{F}$ en fonction de la capacité de cet isolat à produire de la lécithinase, comme le démontre la gélose au jaune d'œuf; son incapacité à produire de la lipase et sa capacité de produire de la toxine de type $\mathrm{F}$ dans le bouillon de culture, détectée à l'aide d'essais biologiques chez la souris. L'API20 A a été utilisé pour confirmer l'identification de C. baratii de type F. Tous les protocoles d'utilisation des animaux ont été approuvés par les comités de soins et d'utilisation des animaux des établissements (Santé Canada ou le Département californien de la santé publique).

\section{Données sur la morbidité}

Les dossiers sur les renseignements cliniques des patients ont été extraits de la Base de données sur les congés des patients (BDCP) pour la période allant de 2005 à 2018 et de la Base de données sur la morbidité hospitalière (BDMH) pour la période allant de 2005 à 2010 de l'Institut canadien d'information sur la santé (ICIS) en interrogeant tous les dossiers actuellement disponibles qui énuméraient le botulisme dans les 10 premiers codes de diagnostic présumés (25). Ces dossiers ont ensuite été jumelés à des dossiers en fonction de l'âge, du sexe, de la date d'admission, de la date de prélèvement de l'échantillon et de la province ou du territoire de résidence. Seuls les cas confirmés en laboratoire ont été inclus dans les analyses des données provenant de la BDCP et de la BDMH.

\section{Approbation déontologique}

L'approbation officielle en matière d'éthique n'était pas requise puisque cette étude utilisait des données sur les soins de santé dépersonnalisées qui ont été obtenues dans le cadre d'une entente avec I'ICIS et nous présentons les résultats sous forme regroupée.

\section{Résultats}

Même si le botulisme est une maladie infectieuse à déclaration obligatoire au Canada depuis 1933, le premier cas de botulisme infantile a été signalé au Canada en 1979 (16), trois ans après que les premiers cas ont été décrits aux États-Unis $(4,5)$. De 1979 à 2019, il y a eu 63 cas confirmés en laboratoire de botulisme infantile au Canada (figure 1), soit une moyenne de 1,6 cas par année. Le Service de référence pour le botulisme de Santé Canada a confirmé 44 cas, tandis que le Centre de contrôle des maladies de la Colombie-Britannique a confirmé 19 cas. Ces cas de botulisme infantile sont également décrits comme un ensemble de données complet et seront appelés " cas confirmés en laboratoire ".

Figure 1 : Nombre de cas de botulisme infantile confirmés en laboratoire au Canada, de 1979 à 2019

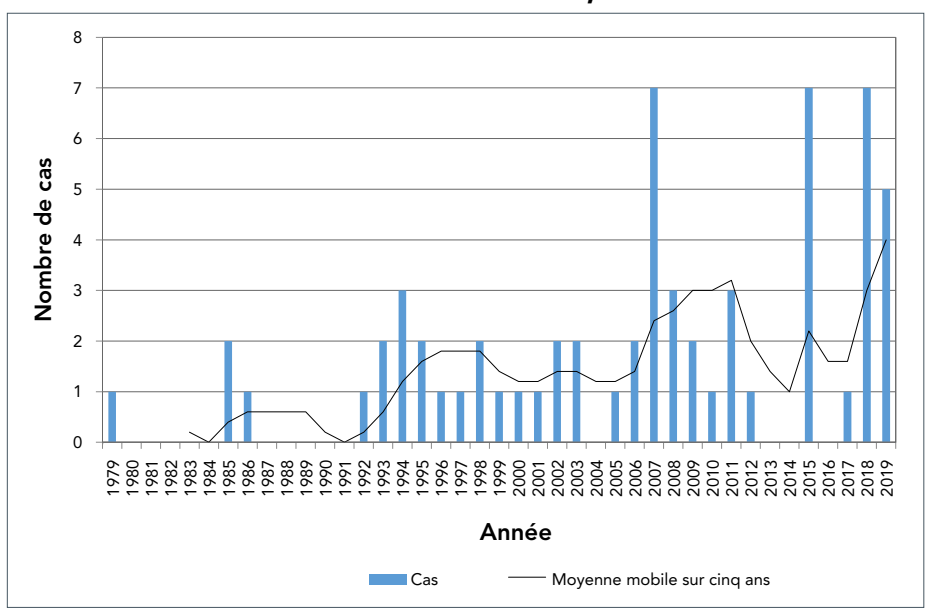

Le botulisme infantile est une maladie rare; l'incidence annuelle moyenne a été calculée à 4,3 cas par million de naissances vivantes au Canada au cours de cette période (tableau 1). Les cas confirmés se sont produits en Ontario $(n=21)$, en Colombie-Britannique $(n=19)$, au Québec $(n=12)$, en Alberta $(n=8)$, à Terre-Neuve-et-Labrador $(n=2)$ et en Nouvelle-Écosse $(n=1)$ (tableau 1). Parmi les 58 dossiers qui indiquaient le sexe, 34 étaient des femmes (59\%) et 24 étaient des hommes (41\%).

\section{Tableau 1 : Cas de botulisme infantile confirmés en laboratoire par sérotype et province et territoire, de 1979 à 2019}

\begin{tabular}{|c|c|c|c|c|c|c|}
\hline \multirow{2}{*}{$\begin{array}{l}\text { Province/ } \\
\text { territoire }\end{array}$} & \multicolumn{4}{|c|}{$\begin{array}{c}\text { Cas par sérotype de } \\
\text { NTBo }\end{array}$} & \multirow{2}{*}{ Total } & \multirow{2}{*}{$\begin{array}{c}\text { Taux annuel/ } \\
\text { million de } \\
\text { naissances } \\
\text { vivantes }^{a}\end{array}$} \\
\hline & $A$ & B & $A B$ & $F$ & & \\
\hline Ontario & 11 & 9 & 1 & 0 & 21 & 3,75 \\
\hline $\begin{array}{l}\text { Colombie- } \\
\text { Britannique }\end{array}$ & 19 & 0 & 0 & 0 & 19 & 10,86 \\
\hline Québec & 9 & 2 & 0 & 1 & 12 & 3,63 \\
\hline Alberta & 8 & 0 & 0 & 0 & 8 & 4,41 \\
\hline $\begin{array}{l}\text { Terre-Neuve- } \\
\text { et-Labrador }\end{array}$ & 1 & 1 & 0 & 0 & 2 & 9,93 \\
\hline $\begin{array}{l}\text { Nouvelle- } \\
\text { Écosse }\end{array}$ & 0 & 1 & 0 & 0 & 1 & 2,66 \\
\hline Canadab & 48 & 13 & 1 & 1 & 63 & 4,30 \\
\hline \multicolumn{7}{|c|}{$\begin{array}{l}\text { Abréviation : NTBo, neurotoxine botulique } \\
\text { a Le taux annuel par million de naissances vivantes a été calculé comme le nombre total de cas } \\
\text { divisé par le nombre moyen de naissances vivantes annuelles de } 1991 \text { à } 2018 \text { multiplié par } 40 \\
\text { (années), divisé par un million }(21,22) \\
\text { b Aucun cas de botulisme infantile confirmé en laboratoire au Manitoba, au Nouveau-Brunswick, } \\
\text { dans les Territoires du Nord-Ouest, au Nunavut, à l'île-du-Prince-Édouard, en Saskatchewan ou } \\
\text { au Yukon }\end{array}$} \\
\hline
\end{tabular}


Par rapport aux cas confirmés en laboratoire, le SCSMDO a signalé 57 cas de botulisme infantile entre 1983 et 2018 , ce qui donne une moyenne de 1,6 cas par année. Compte tenu du cas unique confirmé en laboratoire en 1979 et des cinq cas confirmés en laboratoire en 2019, le nombre total de cas signalés au SCSMDO correspond au nombre total de 57 cas confirmés en laboratoire de 1983 à 2018 . Toutefois, le nombre de cas signalés par les provinces et les territoires variait. Des cas ont été signalés au SCSMDO en Ontario $(n=22)$, en Colombie-Britannique ( $n=14)$, au Québec $(n=10)$, en Alberta $(n=9)$, à Terre-Neuve-et-Labrador $(n=1)$ et en Nouvelle-Écosse $(n=1)$. Sur les 57 cas, 36 étaient des femmes et 21 des hommes. De 1983 à 2018, les cas confirmés en laboratoire provenaient de l'Ontario $(n=19)$, de la Colombie-Britannique $(n=17)$, du Québec $(n=11)$, de l'Alberta $(n=8)$, de Terre-Neuve-et-Labrador $(n=2)$ et de la Nouvelle-Écosse $(n=1)$.

L'âge d'apparition des symptômes suit une distribution unimodale avec une moyenne de 17,8 semaines et une médiane de 16 semaines (figure 2). Le plus jeune nourrisson avait deux semaines et le plus âgé avait 52 semaines. La NTBo de type $A$ constituait la majorité des cas $(n=48 ; 76 \%)$, suivi du type $B(n=13 ; 21 \%)$, du type $A B(n=1 ; 2 \%)$ et type $F$ $(n=1 ; 2 \%)$ (tableau 1$)$. Le cas unique de type $F$ a été identifié comme étant causé par $C$. baratii; c'était le plus jeune nourrisson qui était âgé de deux semaines au moment de l'apparition des symptômes. Toutes les souches de $C$. botulinum de type $A$ sont du groupe I (protéolytique), tandis que les souches de type B peuvent être soit du groupe I, soit du groupe II (non protéolytique). Parmi les souches de type $B$ isolées et analysées pour l'activité protéolytique, toutes les souches $(n=10)$ ont été classées comme étant $C$. botulinum du groupe I.

Figure 2 : Âge d'apparition des symptômes pour les cas de botulisme infantile confirmés en laboratoire au Canada, de 1979 à 2019

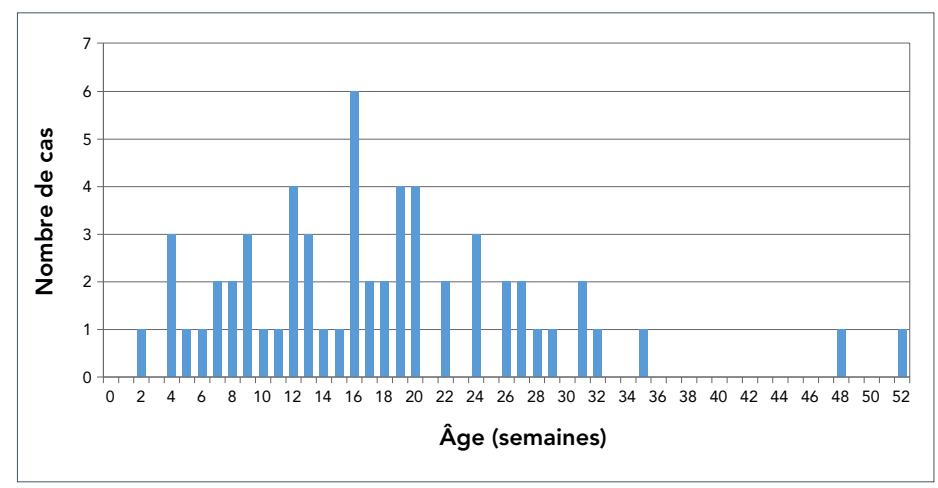

Parmi les 63 cas recensés entre 1979 et 2019, 29 (46\%) ont présenté des échantillons environnementaux ou alimentaires aux fins d'analyse et seulement six ont été jumelés à des échantillons environnementaux, y compris du miel $(n=4)$ et de la poussière sur le berceau ( $n=1)$, ainsi qu'un prélèvement d'échantillons à l'aide d'écouvillons sur le matelas pour changer les couches et un prélèvement d'échantillons à l'aide d'écouvillons dans la baignoire $(n=1)$ (tableau 2). Des 58 dossiers disponibles, les selles étaient de loin le meilleur échantillon clinique pour la détection de la NTBo $(n=55 ; 95 \%)$ et de $C$. botulinum viable ( $n=58 ; 100 \%$ ) (tableau 3 ). La NTBo n'a été détectée que dans trois des 33 échantillons de sérum (9\%) analysés, tandis que ni la NTBo ni le $C$. botulinum n'ont été détectés dans trois échantillons du contenu gastrique analysés.

\section{Tableau 2 : Attribution de la source confirmée en laboratoire du botulisme infantile}

\begin{tabular}{|l|l|l|}
\hline Année & Province/territoire & \multicolumn{1}{|c|}{ Source } \\
\hline 1985 & Québec & Miel \\
\hline 1993 & Alberta & Miel \\
\hline 1995 & Québec & Miel \\
\hline 2000 & Colombie-Britannique & Miel \\
\hline 2005 & Ontario & Poussière sur le berceau \\
\hline 2009 & Québec & $\begin{array}{l}\text { Prélèvement d'échantillons à } \\
\text { I'aide d'écouvillons sur le matelas } \\
\text { pour changer les couches et } \\
\text { prélèvement d'échantillons à l'aide } \\
\text { d'écouvillons dans la baignoire }\end{array}$ \\
\hline
\end{tabular}

Tableau 3 : Détection en laboratoire de la NTBo et de Clostridium botulinum viable dans des échantillons cliniques

\begin{tabular}{l|r|r|r|r|r|}
\multirow{2}{*}{ Échantillon } & \multirow{2}{*}{$\begin{array}{c}\text { Analysé } \\
\text { (n) }\end{array}$} & \multicolumn{2}{|c|}{ NTBo détecté } & \multicolumn{2}{|c|}{$\begin{array}{c}\text { C. botulinum } \\
\text { détecté }\end{array}$} \\
\cline { 3 - 6 } & $\mathbf{n}$ & \multicolumn{1}{c|}{$\begin{array}{r}\text { n } \\
\%\end{array}$} \\
\hline $\begin{array}{l}\text { Contenu } \\
\text { gastrique }\end{array}$ & 3 & 0 & 0 & 0 & 0 \\
\hline Sérum & 33 & 3 & 9 & 0 & 0 \\
\hline Selles & 58 & 55 & 95 & 58 & 100 \\
\hline
\end{tabular}

Des 63 cas de botulisme infantile confirmés en laboratoire, 23 ont été recoupés avec la BDCP pour la période allant de 2005 à 2018 et la BDMH) pour la période allant de 2005 à 2010. La durée moyenne (écart-type [ET]) du séjour à l'hôpital de ces patients était de $20,3(7,0)$ jours (tableau 4). Plus de la moitié $(n=13)$ ont été transférés dans des unités de soins spéciaux. Ces unités de soins spéciaux sont spécialement conçues, dotées de personnel et équipées pour l'observation et le traitement continus des patients qui ne peuvent pas être pris en charge dans une unité générale de soins aigus. II s'agit notamment des unités de soins intensifs et des unités de soins courants (25). Les patients ont passé en moyenne (ET) $12,2(6,3)$ jours dans une unité de soins spéciaux; huit ont eu besoin d'un respirateur (tableau 4). La plupart des patients ont été renvoyés à la maison sans soutien $(n=9)$ ou avec le soutien des préposés aux soins à domicile $(n=10)$. Trois ont été transférés aux soins aigus pendant l'hospitalisation; les 
dispositions pour le congé d'un patient étaient inconnues (tableau 5). Aucun décès n'a été signalé pour les 23 cas examinés.

Tableau 4 : Paramètres hospitaliers pour les cas d'hospitalisation confirmés en laboratoire $(n=23)$

\begin{tabular}{|l|r|r|r|}
\hline \multicolumn{1}{|c|}{$\begin{array}{c}\text { Mesure } \\
\text { hospitalière }\end{array}$} & \multicolumn{1}{|c|}{ Moyenne } & \multicolumn{1}{c|}{ Écart-type } & \multicolumn{2}{c|}{$\begin{array}{c}\text { Nombre de } \\
\text { cas }\end{array}$} \\
\hline $\begin{array}{l}\text { Durée du séjour } \\
\text { (jours) }\end{array}$ & 20,3 & 7,0 & 23 \\
\hline $\begin{array}{l}\text { Temps passé en } \\
\text { soins spéciaux } \\
\text { (jours) }\end{array}$ & 12,2 & 6,3 & 13 \\
\hline $\begin{array}{l}\text { Utilisation du } \\
\text { respirateur }\end{array}$ & s.o. & s.o. & \\
\hline
\end{tabular}

Tableau 5 : Résultats cliniques des cas d'hospitalisation confirmés en laboratoire $(n=23)$

\begin{tabular}{|l|r|}
\hline \multicolumn{1}{|c|}{ Dispositions pour le congé } & \multicolumn{2}{|c|}{ Nombre de cas } \\
\hline À la maison, pas de soutien & 9 \\
\hline À la maison avec soutien & 10 \\
\hline Transféré aux soins aigus & 3 \\
\hline Inconnue & 1 \\
\hline Décès & 0 \\
\hline
\end{tabular}

\section{Discussion}

De 1979 à 2019, il y a eu 63 cas confirmés en laboratoire de botulisme infantile au Canada. De 1983 à 2018, 57 cas ont été signalés au SCSMDO, ce qui correspondait au nombre total de cas confirmés en laboratoire au cours de cette période. Toutefois, les provinces et les territoires ayant signalé les cas différaient. Les raisons des écarts sont inconnues. Toutefois, cette divergence n'est pas inattendue, car les laboratoires de référence participent directement au diagnostic des cas, tandis que les autorités de santé publique peuvent ne pas être impliquées dans chaque cas sporadique. Au cours des dernières années, le Service de référence pour le botulisme pour le Canada et l'Agence de la santé publique du Canada ont collaboré afin d'assurer des rapports plus complets aux autorités compétentes en matière de santé publique.

Le taux d'incidence annuel de 4,3 cas par million de naissances vivantes au Canada est semblable à celui signalé en Australie (4,4 cas par million de naissances vivantes), en Italie $(2,1$ cas par million de naissances vivantes) et au Danemark (6,7 cas par million de naissances vivantes) et inférieur aux taux déclarés aux États-Unis (20,3 cas par million de naissances vivantes) et en Argentine (24,1 cas par million de naissances vivantes) (26).
L'âge moyen à l'apparition des symptômes était de 17,8 semaines au Canada. Ce nombre est supérieur à l'âge moyen déclaré de 13,8 semaines aux États-Unis et de 14,3 semaines dans le monde à l'extérieur des États-Unis (26). L'âge d'apparition des symptômes peut correspondre à des changements dans le microbiote intestinal après le sevrage (27) qui rend l'intestin plus sensible à la colonisation. Une étude récente a révélé que les patients atteints de botulisme infantile nourris au sein étaient plus âgés à l'apparition des symptômes que les patients nourris au lait maternisé (28), même si le rôle de l'allaitement maternel dans le botulisme infantile soit controversé $(29,30)$.

La conclusion selon laquelle $98 \%$ des cas étaient des types $A, B$ ou $A B$ est conforme à un rapport selon lequel les types $A$ et $B$ de C. botulinum représentaient $98,7 \%$ de tous les cas enregistrés de botulisme infantile dans le monde de 1978 à 2006 (26). Le cas unique de type $\mathrm{F}$ identifié comme étant causé par $C$. baratii est rare, mais non sans précédent. Des cas de botulisme infantile causé par des souches toxigènes de $C$. butyricum de type $E(8-10)$ et de $C$. baratii de type $F(11-14)$, ainsi qu'un cas unique causé par $C$. botulinum de type $E(31)$, ont été décrits ailleurs. La prédominance de $C$. botulinum de type $A$ dans l'ouest des États-Unis est corrélée par la présence de cas de type $A$ seulement dans les deux provinces de l'Ouest, soit l'Alberta et la Colombie-Britannique (tableau 1) (32).

La nature sporadique du botulisme infantile et l'ubiquité des spores de $C$. botulinum dans l'environnement, combinée à ce qui est probablement une faible dose infectieuse, font de la détection de la source de $C$. botulinum un défi important. En revanche, les éclosions de botulisme d'origine alimentaire ont tendance à comporter de multiples cas, permettant des enquêtes épidémiologiques et la détermination de sources alimentaires potentielles. Pour les 29 cas où des échantillons environnementaux ou alimentaires ont été présentés aux fins d'analyses, l'isolement de $C$. botulinum était un cas rare. Le miel a compté pour quatre cas, en fonction de l'isolement du même sérotype provenant du miel donné au nourrisson. Dans un cas, $C$. botulinum a été isolé dans un échantillon de poussière provenant du berceau. Dans un autre cas, un matelas pour changer les couches et une baignoire se sont révélés positifs pour $C$. botulinum. Dans ces cas, il est impossible de savoir si les échantillons environnementaux étaient la source de la maladie chez le nourrisson ou s'ils étaient contaminés par l'excrétion provenant des selles de ce dernier. Un cas de botulisme infantile en Finlande a été lié à des spores de $C$. botulinum isolées dans de la poussière provenant d'un aspirateur (33). L'absence d'antécédents de consommation de miel ne devrait pas être considérée comme une raison pour exclure le botulisme infantile (18).

Pour la détection en laboratoire de la NTBo ou l'isolement de $C$. botulinum viable, le prélèvement et la manipulation appropriés des échantillons cliniques sont essentiels. Des 
58 dossiers disponibles, les selles était de loin le meilleur échantillon clinique pour la détection de la NTBo (95\%) et de C. botulinum viable (100\%). Cela est conforme à un rapport précédent selon lequel les effluents de selles ou de lavements sont des échantillons de diagnostic préférables pour le botulisme infantile (34).

Des 23 cas de botulisme infantiles qui ont été comparés à la $\mathrm{BDCP}$ et à la $\mathrm{BDMH}, 13$ (57\%) ont été transférés dans des unités de soins spéciaux et huit ( $35 \%$ ) ont eu besoin du soutien d'un respirateur. Aucun décès n'a été signalé. Cela est conforme aux données antérieures indiquant que le botulisme infantile est une maladie grave nécessitant une intervention dans la majorité des cas. À l'extérieur des États-Unis, le pourcentage de cas signalés qui nécessitaient le soutien d'un respirateur ou une intubation était de $67 \%$ et les décès représentaient $1,1 \%$ des cas (26). Aux États-Unis, 56 \% des cas de botulisme infantile hospitalisé ont nécessité un soutien au moyen d'un respirateur ou une intubation pendant un essai de BabyBIG (15), alors que le taux de mortalité aux États-Unis est inférieur à 1 \% (35).

\section{Limites}

La gamme restreinte de dossiers hospitaliers disponibles dans les bases de données de l'ICIS (de 2005 à 2018 pour la BDCP et de 2005 à 2010 pour la BDMH) a réduit le nombre de cas qui pouvait être lié aux dossiers de laboratoire et a réduit le pouvoir statistique des données obtenues des dossiers hospitaliers, comme la gravité de la maladie et l'efficacité du traitement. Il convient de noter que le traitement à l'aide de l'antitoxine BabyBIG n'est pas systématiquement consigné dans la base de données de I'ICIS; nous recommandons que cela soit inclus pour évaluer correctement l'effet de ce traitement.

De même, 28 cas de la base de données de l'ICIS ont été codés comme " botulisme » en fonction d'un diagnostic différentiel, mais les échantillons n'ont pas été envoyés à un laboratoire aux fins de confirmation. Enfin, il y a eu plusieurs cas de botulisme infantile confirmés en laboratoire qui n'ont pas pu être identifiés dans les bases de données de I'ICIS, même au cours des années mentionnées. En tenant compte d'un récent résumé de 1345 cas de botulisme infantile en Californie pour la période allant de 1976 à 2016, il est possible de constater que 99,3\% des cas ont été hospitalisés (36), il est probable que les dossiers hospitaliers manquants pour les cas confirmés en laboratoire au Canada étaient le résultat d'un code de diagnostic manquant dans la base de données de I'ICIS. Une meilleure communication entre les hôpitaux, les responsables de la santé publique et les laboratoires de diagnostic aiderait à obtenir tous les cas de botulisme infantile au Canada et aiderait à diagnostiquer et à évaluer les traitements. Les médecins et le personnel hospitalier peuvent consulter le Guide canadien de botulisme à l'intention des professionnels de la santé pour obtenir des directives sur le diagnostic différentiel, le prélèvement approprié des échantillons cliniques et le traitement avec l'antitoxine BabyBIG (37).

\section{Conclusion}

Le botulisme infantile est une maladie rare au Canada. La distribution unimodale de l'âge d'apparition des symptômes suggère une sensibilité temporelle à la colonisation par C. botulinum qui atteint un sommet à 16 semaines. Même si aucun décès n'a été signalé au cours de cette période, la durée moyenne du séjour à l'hôpital et le nombre de cas transférés à des soins spéciaux démontrent de graves résultats cliniques pour les patients atteints de cette maladie. L'efficacité de l'antitoxine BabyBIG n'a pas pu être évaluée en raison des limites de la déclaration et devrait être traitée à l'avenir.

\section{Déclaration des auteurs}

R. H. - Examen des dossiers de laboratoire du Service de référence sur le botulisme de Santé Canada; les dossiers consultés dans la Base de données sur les congés des patients et dans la Base de données sur la morbidité hospitalière de l'Institut canadien d'information sur la santé pour obtenir des données sur l'hospitalisation de cas de botulisme infantile; a effectué toutes les analyses statistiques; a effectué des recherches documentaires supplémentaires; a rédigé la première ébauche C. T. - Examen des dossiers de laboratoire du laboratoire de microbiologie de santé publique de la Colombie-Britannique; a commenté sur le manuscrit approuvé

N. P. - Examen des dossiers de laboratoire du laboratoire de microbiologie de santé publique de la Colombie-Britannique; a commenté sur le manuscrit approuvé

J. C. - A interrogé le Système canadien de surveillance des maladies à déclaration obligatoire pour obtenir des données sur les cas signalés de botulisme infantile, a rédigé la section sur le Système canadien de surveillance des maladies et a fait des commentaires sur l'approbation du manuscrit J. A. - A conçu le résumé, examiné les dossiers de laboratoire du Service de référence sur le botulisme de Santé Canada, a effectué des recherches documentaires supplémentaires, a rédigé certaines sections et a révisé l'article

\section{Intérêts concurrents}

Aucun.

\section{Remerciements}

Les auteurs tiennent à remercier J. Barash et $\mathrm{S}$. Arnon du Programme de traitement et de prévention du botulisme infantile du Département de la santé publique de Californie pour I'isolement et l'identification de $C$. baratii type $F$ comme agent causal du cas unique de botulisme infantile de type $F$ dans un échantillon de selles envoyé au Programme de traitement et de prévention du botulisme infantile du Département de la santé publique de Californie à des fins de recherche. 


\section{Financement}

Ce travail a été appuyé par Santé Canada, le laboratoire de santé publique du Centre de contrôle des maladies de la Colombie-Britannique et l'Agence de la santé publique du Canada.

\section{Références}

1. Fleck-Derderian S, Shankar M, Rao AK, Chatham-Stephens K, Adjei S, Sobel J, Meltzer MI, Meaney-Delman D, Pillai SK. The epidemiology of foodborne botulism outbreaks: a systematic review. Clin Infect Dis 2017;66(suppl_1):S73-81. DOI

2. Chatham-Stephens K, Fleck-Derderian S, Johnson SD, Sobel J, Rao AK, Meaney-Delman D. Clinical features of foodborne and wound botulism: a systematic review of the literature, 1932-2015. Clin Infect Dis 2017;66(suppl_1):S11-6. DOI

3. Harris RA, Anniballi F, Austin JW. Adult intestinal toxemia botulism. Toxins (Basel) 2020;12(2):81. DOI PubMed

4. Pickett J, Berg B, Chaplin E, Brunstetter-Shafer MA. Syndrome of botulism in infancy: clinical and electrophysiologic study. N Engl J Med 1976;295(14):770-2. DOI PubMed

5. Midura TF, Arnon SS. Infant botulism. Identification of Clostridium botulinum and its toxins in faeces. Lancet 1976;308(7992):934-6. DOI PubMed

6. Arnon SS, Damus K, Chin J. Infant botulism: epidemiology and relation to sudden infant death syndrome. Epidemiol Rev 1981;3:45-66. DOI PubMed

7. Arnon SS. Infant botulism. Annu Rev Med 1980;31:541-60. DOI PubMed

8. Fenicia L, Da Dalt L, Anniballi F, Franciosa G Zanconato S, Aureli P. A case if infant botulism due to neurotoxigenic Clostridium butyricum type $\mathrm{E}$ associated with Clostridium difficile colitis. Eur J Clin Microbiol Infect Dis 2002;21(10):736-8. DOI PubMed

9. McCroskey LM, Hatheway CL, Fenicia L, Pasolini B, Aureli P. Characterization of an organism that produces type $\mathrm{E}$ botulinal toxin but which resembles Clostridium butyricum from the feces of an infant with type $E$ botulism. J Clin Microbiol 1986;23(1):201-2. DOI PubMed

10. Aureli P, Fenicia L, Pasolini B, Gianfranceschi M, McCroskey LM, Hatheway CL. Two cases of type $E$ infant botulism caused by neurotoxigenic Clostridium butyricum in Italy. J Infect Dis 1986;154(2):207-11. DOI PubMed

11. Paisley JW, Lauer BA, Arnon SS. A second case of infant botulism type $\mathrm{F}$ caused by Clostridium baratii. Pediatr Infect Dis J 1995;14(10):912-4. DOI PubMed
12. Barash JR, Tang TW, Arnon SS. First case of infant botulism caused by Clostridium baratii type $\mathrm{F}$ in California. J Clin Microbiol 2005;43(8):4280-2. DOI PubMed

13. Halpin AL, Khouri JM, Payne JR, Nakao JH, Cronquist A, Kalas N, Mohr M, Osborne M, O’Dell S, Luquez C, Klontz KC, Sobel J, Rao AK. Type F infant botulism: Investigation of recent clusters and overview of this exceedingly rare disease. Clin Infect Dis 2017;66(suppl_1):S92-4. DOI

14. Moodley A, Quinlisk P, Garvey A, Kalas N, Barash JR, Khouri JM; Centers for Disease Control and Prevention (CDC). Notes from the field: infant botulism caused by Clostridium baratii type F - lowa, 2013. MMWR Morb Mortal Wkly Rep 2015;64(14):400. PubMed

15. Arnon SS, Schechter R, Maslanka SE, Jewell $N P$, Hatheway CL. Human botulism immune globulin for the treatment of infant botulism. N Engl J Med 2006;354(5):462-71. DOI PubMed

16. McCurdy DM, Krishnan C, Hauschild AH. Infant botulism in Canada. Can Med Assoc J 1981;125(7):741-3. PubMed

17. Roland EH, Ebelt VJ, Anderson JD, Hill A. Infant botulism: a rare entity in Canada? CMAJ 1986;135(2):130-1. PubMed

18. Siu K, Rehan M, Austin JW, Ramachandran Nair R, Pernica J. It's not all about the honey. Paediatr Child Health 2017;22(1):37-8. DOI PubMed

19. Hauschild AH, Bowmer EJ, Gauvreau L. Infant botulism. Can Med Assoc J 1978;118(5):484. PubMed

20. Schwartz KL, Austin JW, Science M. Constipation and poor feeding in an infant with botulism. CMAJ 2012;184(17):1919-22. DOI PubMed

21. Statistique Canada. Profil du recensement, Recensement de 2016 [Internet]. Ottawa (ON) : Statistique Canada; (modifié 2021-03-11; accédé 2020-09-28). https://www12. statcan.gc.ca/census-recensement/2016/dp-pd/prof/index. $\mathrm{cfm}$ ?Lang $=\mathrm{F}$

22. United Nations Statistics Division. UNdata [Internet]. New York (NY): UNSD; (accédé 2020-09-28). http://data.un.org/fr/ index.html

23. Définition nationale de cas : Botulisme [Internet]. Ottawa (ON) : Gouvernement du Canada; (modifié 2019-11-26; accédé 2020-12-22). https://www. canada.ca/fr/sante-publique/services/maladies/botulisme/ professionnels/definition-nationale-cas.html

24. Austin J, Sanders G. Méthodes de la DGPS pour l'analyse microbiologique des aliments, Volume 2: Détection de Clostridium botulinum et de ses toxines dans des aliments suspects et dans des prélèvements cliniques. 2009 (modifié 2018-05-09; accédé 2020-12-22). https://www. canada.ca/fr/sante-canada/services/aliments-nutrition/ programmes-recherche-methodes-analyse/methodesanalyse/compendium-methodes/methodes-dgps-analysemicrobiologique-aliments-compendium-methodes.html 
25. Institut canadien d'information sur la santé. Tendances des hospitalisations et de la durée moyenne du séjour au Canada, 2003-2004 et 2004-2005. Analyse en bref. Ottawa (ON) : ICIS; 2005. https://secure.cihi.ca/free_products/ hmdb_analysis_in_brief_f.pdf

26. Koepke R, Sobel J, Arnon SS. Global occurrence of infant botulism, 1976-2006. Pediatrics 2008;122(1):e73-82.

DOI PubMed

27. Leong C, Haszard JJ, Lawley B, Otal A, Taylor RW, Szymlek-Gay EA, Fleming EA, Daniels L, Fangupo LJ, Tannock GW, Heath AM. Mediation analysis as a means of identifying dietary components that differentially affect the fecal microbiota of infants weaned by modified baby-led and traditional approaches. Appl Environ Microbiol 2018;84(18):e00914-8. DOI PubMed

28. Panditrao MV, Dabritz HA, Kazerouni NN, Damus KH, Meissinger JK, Arnon SS. Seven-year case-control study in California of risk factors for infant botulism. J Pediatr 2020;227:258-267.e8. DOI PubMed

29. Arnon SS, Damus K, Thompson B, Midura TF, Chin J. Protective role of human milk against sudden death from infant botulism. J Pediatr 1982;100(4):568-73.

DOI PubMed

30. Spika JS, Shaffer N, Hargrett-Bean N, Collin S, MacDonald KL, Blake PA. Risk factors for infant botulism in the United States. Am J Dis Child 1989;143(7):828-32.

DOI PubMed
31. Lúquez C, Dykes JK, Yu PA, Raphael BH, Maslanka SE. First report worldwide of an infant botulism case due to Clostridium botulinum type $\mathrm{E}$. J Clin Microbiol 2010;48(1):326-8. DOI PubMed

32. Smith LD. The occurrence of Clostridium botulinum and Clostridium tetani in the soil of the United States. Health Lab Sci 1978;15(2):74-80. PubMed

33. Nevas $M$, Lindström $M$, Virtanen A, Hielm S, Kuusi M, Arnon SS, Vuori E, Korkeala H. Infant botulism acquired from household dust presenting as sudden infant death syndrome. J Clin Microbiol 2005;43(1):511-3. DOl PubMed

34. Midura TF. Update: infant botulism. Clin Microbiol Rev 1996;9(2):119-25. DOI PubMed

35. Jackson KA, Mahon BE, Copeland J, Fagan RP. Botulism mortality in the USA, 1975-2009. Botulinum J 2015;3(1):6-17. DOI PubMed

36. Panditrao MV, Dabritz HA, Kazerouni NN, Damus KH, Meissinger JK, Arnon SS. Descriptive epidemiology of infant botulism in California: the first 40 years. J Pediatr 2020;227:247-257.e3. DOI PubMed

37. Santé Canada. Botulisme - Guide pour les professionnels de la santé [Internet]. Ottawa (ON) : Santé Canada; 2020 (accédé 2020-12-22). https://www.canada.ca/fr/santecanada/services/aliments-nutrition/legislation-lignesdirectrices/document-reference/botulisme-guideprofessionnels-sante-2012.html 